\section{Associação entre estado nutricional da mãe e a frequência, local e companhia durante as refeições com o sobrepeso/obesidade de adolescentes da cidade de Florianópolis, Brasil}

\section{The association between nutritional status of the mother and the frequency and location of and company during meals and overweight/obesity among adolescents in the city of Florianópolis, Brazil}

\begin{abstract}
Objectives: to estimate the prevalence of overweight/obesity and to investigate the association between nutritional status of the mother, socio-demographic factors, frequency and location of and company during meals and overweight/obesity in schoolchildren aged between 11 and 14 years in Florianópolis, Santa Catarina.

Methods: a cross-sectional study was carried out with 962 adolescents attending public and private schools. Overweight/obesity was defined according to the World Health Organization criteria. The independent variables were: school network, sex of schoolchild, level of schooling and nutritional status of mother, monthly household income, frequency and location of and company during meals. Raw and adjusted analyses were conducted using the Poisson regression to estimate prevalence rates with confidence intervals of $95 \%$.

Results: the prevalence of overweight/obesity was 29.8\% (CI95\%: 25.7-33.9), 11.6\% for obesity and $18.3 \%$ for overweight. Among students in the public network, overweight/obesity was associated with overweight/obesity in the mother, more breakfasts, having lunch at school or in another location, and having breakfast with others, while, among private network students, it was associated only with overweight/obesity in the mother.
\end{abstract}

Conclusions: the high prevalence of overweight/obesity, both in the public and the private network, demonstrates the need to pay more attention to the associated factors, such as overweight/obesity in the mother, frequency and location of and company during meals.

Key words Adolescent, Overweight, Obesity, Food consumption, Meals
Gisele Liliam D'Avila 1

Roberta Luisa Müller 2

Priscila Schramm Gonsalez 3

Francisco de Assis Guedes de Vasconcelos 4

1-4 Departamento de Nutrição. Universidade Federal de Santa Catarina. Campus Universitário Trindade. Florianópolis, SC, Brasil. CEP: 88.040-900. E-mail: f.vasconcelos@ufsc.br

\section{Resumo}

Objetivos: estimar a prevalência de sobrepeso/obesidade e analisar a associação entre estado nutricional da mãe, fatores sociodemográficos, frequência, local e companhia nas refeições e sobrepeso/obesidade em escolares de 11 a 14 anos de Florianópolis, Santa Catarina.

Métodos: estudo transversal com 962 adolescentes de escolas públicas e privadas. O sobrepeso/obesidade foi definido de acordo com a Organização Mundial da Saúde. As variáveis independentes foram: rede de ensino, sexo do escolar, escolaridade e estado nutricional da mãe, renda familiar mensal, frequência, local e companhia nas refeições. Foram realizadas análises brutas e ajustadas por meio da regressão de Poisson estimando-se razões de prevalência e intervalos de confiança de $95 \%$.

Resultados: a prevalência de sobrepeso/obesidade foi de 29,8\% (IC95\%: 25,7-33,9), sendo 11,6\% de obesidade e 18,3\% de sobrepeso. Entre alunos da rede pública, o sobrepeso/obesidade esteve associado ao sobrepeso/obesidade da mãe, maior número de realização de café da manhã, almoçar na escola ou em outro local e café da manhã na companhia de outras pessoas, enquanto que entre estudantes da rede privada, o evento esteve associado ao sobrepeso/obesidade da mãe.

Conclusão: a elevada prevalência de sobrepeso/obesidade, tanto na rede pública quanto na privada, evidencia a necessidade de mais atenção para os fatores associados, tais como sobrepesolobesidade da mãe, frequência, local $e$ companhia nas refeições.

Palavras-chave Adolescente, Sobrepeso, Obesidade, Consumo de alimentos, Refeições 


\section{Introdução}

A obesidade na adolescência constitui um evento cuja determinação é complexa e multifatorial, envolvendo a participação de fatores biológicos e ambientais. ${ }^{1}$ A literatura tem apontado evidências científicas sobre a associação entre fatores comportamentais, tais como frequência, local e companhia na realização das refeições, ${ }^{2-4} \mathrm{e}$ fatores sociodemográficos, tais como sexo, escolaridade e estado nutricional da mãe e renda mensal familiar5-7 e obesidade na adolescência.

A família agrega um conjunto de valores, crenças, conhecimentos e hábitos que podem influenciar práticas de promoção da saúde ou da doença de seus membros. Por isso, a dinâmica familiar assume papel imprescindível na mudança de práticas alimentares de seus filhos para controle ou tratamento da obesidade, 8,9 pondo em destaque a importância da realização das refeições em companhia dos pais como fator de prevenção da obesidade e na promoção de hábitos alimentares saudáveis. 4,10

Estudos sobre prevalência e fatores associados ao sobrepeso/obesidade em adolescentes têm tido destaque nas últimas décadas.11-13 Entretanto, informações sobre a associação entre frequência, local e companhia nas refeições e sobrepeso/obesidade em escolares de 11 a 14 anos de idade ainda são escassos. 6,10,14 Estudos prévios mostraram associação entre o consumo habitual de café da manhã, a presença dos pais nas refeições reduziu o risco de sobrepeso/obesidade. 10,14

O objetivo do presente artigo foi estimar a prevalência de sobrepeso/obesidade e analisar a sua associação com o estado nutricional da mãe, fatores sociodemográficos, frequência, local e companhia durante o consumo das refeições e sobrepeso/obesidade em escolares de 11 a 14 anos de idade de escolas públicas e privadas de Florianópolis, Santa Catarina.

\section{Métodos}

Estudo de corte seccional realizado em uma amostra aleatória de escolares de 11 a 14 anos de idade matriculados em escolas públicas e privadas de Florianópolis, Santa Catarina. O estudo é parte de uma pesquisa de maior abrangência, conduzida com o objetivo de analisar a prevalência e fatores associados à obesidade em escolares de sete a 14 anos de idade. Detalhes dos procedimentos metodológicos foram descritos em estudo anterior. 15

O universo do qual a amostra foi selecionada era composto de 26.075 alunos do ensino fundamental, de 11 a 14 anos de idade, matriculados em 85 escolas, dos turnos matutino e vespertino, de acordo com censo escolar de 2010. O tamanho da amostra foi calculado com base nos seguintes parâmetros: prevalência esperada de $38 \%$ de sobrepeso/obesidade, 6,16 erro amostral de $5 \%$, intervalo de confiança de $95 \%$, efeito de desenho de 1,8 e percentual de perdas de $10 \%$, obtendo-se um $n=707$ escolares de 11 a 14 anos de idade.

A amostragem foi por conglomerados, tendo as escolas sido divididas em dez estratos de acordo com as regiões político-administrativas do município de Florianópolis (Centro, Continente, Norte, Leste e Sul) e natureza da escola (pública ou privada). Selecionou-se aleatoriamente 30 escolas (19 públicas e 11 privadas) em cada estrato. Em seguida, a seleção das turmas em cada escola foi realizada de forma sistemática com base na listagem dos escolares matriculados. Em cada escola, foram sorteadas duas turmas, uma do turno matutino e outra do vespertino.

A coleta de dados foi feita entre setembro de 2012 a junho de 2013 por uma equipe treinada para padronização das medidas antropométricas. 17 Foi realizado estudo piloto para uniformização dos procedimentos de coleta e averiguação da aplicabilidade dos instrumentos.

Dados maternos sobre nível de escolaridade (nunca estudou, ensino fundamental, ensino médio e ensino superior) e estado nutricional (definido com base nos dados autorreferidos de peso e altura) foram obtidos por meio de um questionário preenchido pelos pais ou responsáveis.

A coleta das informações sobre frequência, local e companhia durante o consumo das refeições (café da manhã, almoço, jantar e lanches) no período de uma semana, foi realizada por meio de questionário autopreenchido pelos adolescentes.

As medidas antropométricas (peso e estatura) para o cálculo do índice de massa corporal (IMC) dos escolares foram realizadas nas escolas, segundo normas internacionais de padronização. $18 \mathrm{O}$ peso foi aferido utilizando balança eletrônica da marca Marte ${ }^{\circledR}$, modelo LC 200 PP, com capacidade mínima de $1 \mathrm{~kg}$ e máxima de $199,95 \mathrm{~kg}$ e precisão de 50 gramas, enquanto que a estatura foi obtida utilizando estadiômetro da marca Alturexata ${ }^{\circledR}$, com precisão de um milímetro.

A variável dependente foi o sobrepeso/obesidade definida como um valor de IMC $>$ escore- $z+1$, de acordo a classificação proposta pela Organização Mundial da Saúde (OMS, 2007). 19

As variáveis independentes foram: rede de 
ensino (pública e privada), sexo do escolar, escolaridade materna ( $<8$ anos; $>8$ anos), estado nutricional materno (sem sobrepeso/obesidade IMC $<25 \mathrm{~kg} / \mathrm{m}^{2} \mathrm{e}$ com sobrepeso/obesidade IMC $\left.>25 \mathrm{~kg} / \mathrm{m}^{2}\right)^{20}$ renda mensal familiar (tercis de distribuição de reais), frequência das refeições ( $<4$ vezes/semana; $>5$ vezes/semana), local (casa; escola/outro local) e companhia (sozinho; pai e/ou mãe; outros acompanhantes).

A frequência da realização das refeições (café da manhã, almoço, jantar e lanches) de acordo com o local foi classificada nas seguintes categorias: "nunca", "1-2 dias", "3-4 dias", "5-6 dias" ou "todos os dias". O local da refeição foi categorizado em: casa, escola, lanchonete "fast food", outro tipo de restaurante ou na residência de outra família. Em relação à companhia durante as refeições, foram consideradas as seguintes opções, de acordo com o proposto por Araki et al.21: sozinho, somente com a mãe, somente com o pai, com ambos os pais, com os avós ou com outras pessoas da família ou amigos, devendo esta última alternativa ser especificada.

Os dados foram digitados em dupla entrada utilizando o programa Epi-Data ${ }^{\circledR}$, versão 3.2 (Epidata Association, Odense, Dinamarca). A análise estatística foi realizada no software STATA $^{\circledR}$ (Statistical Software for Professionals, Texas), versão 11.0. Em todas as análises foi utilizado o comando $S V Y$.

A análise foi estratificada de acordo com a rede de ensino (pública e privada), tendo em vista as características distintas de ambos os grupos em relação aos determinantes do sobrepeso/obesidade, segundo estudos prévios.6,7,11,15,22,23 Inicialmente realizou-se a análise descritiva das características da população de estudo, tendo as diferenças entre os grupos (pública ou privada) sido testadas pelo teste do qui-quadrado.

A prevalência de sobrepeso/obesidade e seus respectivos intervalos de confiança de $95 \%$ foram estimados de acordo com a rede de ensino (pública e privada). As análises brutas e ajustadas da associação entre o sobrepeso/obesidade e as variáveis foram realizadas utilizando o modelo de regressão de Poisson de acordo com a rede de ensino. Na análise ajustada convencional foram incluídas todas as variáveis independentes e ajustadas conjuntamente, utilizando-se o método para trás (backward), em seguida foram mantidas no modelo final da análise as variáveis que apresentaram $p<0,20$. Os resultados são apresentados pelas razões de prevalências e respectivos intervalos de confiança de $95 \%$. Na interpretação e análise dos resultados das associações considerou-se uma significância de 5\%, ou seja, $p<0,05$.

O estudo foi aprovado pelo Comitê de Ética em Pesquisa com Seres Humanos da Universidade Federal de Santa Catarina (parecer $n^{\circ} 120341 / 2012$ ) conforme normas estabelecidas pela Resolução 466/2012 do Conselho Nacional de Saúde.

\section{Resultados}

Do total de 1848 escolares elegíveis, 975 (52,8\%) dos escolares tiveram o consentimento dos pais para participarem do estudo (52,8\% dos elegíveis), 873 $(47,2 \%)$ se recusaram e 13 foram excluídos por falta de dados antropométricos completos, totalizando uma amostra de 962 adolescentes.

A análise comparativa das características da população que participou do estudo, em relação a que não participou, não constatou diferenças de acordo com série escolar $(p=0,464)$. Houve diferença em relação à idade $(p<0,001)$, ao sexo $(p<0,001)$ e à rede de ensino $(p<0,001)$. Entretanto, o número de participantes alcançou o tamanho da amostra calculado $(\mathrm{n}=707)$.

A Tabela 1 apresenta a análise comparativa das características dos participantes de acordo com rede de ensino (pública versus privada). Observou-se maior frequência de mães com mais de oito anos de estudo na rede privada (61,6\% versus $95,7 \%$; $p<0,001$ ), enquanto que a frequência de sobrepeso/obesidade das mães foi maior entre escolares da rede pública $(52,2 \%$ versus $33,0 \%$; $p<0,001)$. Houve maior frequência de escolares com categoria de renda mensal familiar mais elevada na escola privada $\left(3^{\circ}\right.$ tercil $\left.=>\mathrm{R} \$ 7.000\right)$, quando comparados aos da rede pública $(74,1 \%$ versus $15,7 \% ; p<0,001)$. Não foram observadas diferenças estatisticamente significantes entre escolares da rede pública e privada, quanto à frequência, local e companhia durante o café da manhã. A rede pública apresentou maior frequência de escolares que realizavam o almoço em casa $(87,5 \%$ versus 79,0 ; $p=0,041)$, sem diferenças significantes quanto à frequência desta refeição.

A prevalência global de sobrepeso/obesidade foi de 29,8\% (IC95\%: 25,7-33,9), sendo 18,3\% de sobrepeso e $11,6 \%$ de obesidade. Entre os escolares da rede pública, a prevalência de sobrepeso/obesidade foi de 31,0\% (IC95\%: 26,4-35,6), enquanto que na rede privada foi de 27,4\% (IC95\%: 18,5-36,3), sem diferença estatisticamente significativa $(p=0,439)$ (Tabela 1$)$.

As Tabelas 2 e 3 apresentam os resultados das análises brutas e ajustadas da associação do sobrepeso/obesidade com os fatores investigados, 
entre estudantes da rede pública e privada, respectivamente.

Na rede pública, observou-se que a prevalência de sobrepeso/obesidade nos escolares cujas mães tinham sobrepeso/obesidade foi 1,5 vezes maior do que nos escolares com mães sem sobrepeso/obesidade $(p=0,019)$. Quanto à frequência semanal de refeições, os escolares que tomavam café da manhã mais do que cinco tiveram $40 \%$ menos chance de apresentar sobrepeso/obesidade quando comparados aos que realizavam a refeição menos de vezes por semana $(p=0,001)$. Com relação à companhia durante este refeição, observou-se que os escolares que realizavam o café da manhã com outras pessoas apresentaram prevalência de sobrepeso/obesidade
2,1 vezes maior que os escolares que realizavam esta refeição sozinho ou na companhia dos pais ( $p=0,016)$, (IC95\%: 1,2-3,9). Quanto ao local do almoço, observou-se que a prevalência de sobrepeso/obesidade entre os que almoçavam na escola ou em outro local foi $60 \%$ menor do que os que realizavam esta refeição em casa $(p=0,22)$.

Entre estudantes da rede privada, o sobrepeso/ obesidade da mãe único fator estatisticamente associado ao sobrepeso/obesidade do escolar foi, tendose observado que escolares de mães com sobrepeso/ obesidade apresentaram prevalência de sobrepeso/ obesidade 2,2 vezes maior do que aqueles cujas mães não apresentavam sobrepeso/obesidade $(p=0,018)$.

Tabela 1

Caracterização da amostra de escolares de 11 a 14 anos de idade, segundo as variáveis independentes, estratificadas por rede de ensino. Florianópolis, SC, Brasil, 2012/2013.

\begin{tabular}{|c|c|c|c|c|c|c|c|}
\hline \multirow[t]{2}{*}{ Variáveis } & \multicolumn{2}{|c|}{ Total } & \multicolumn{2}{|c|}{ Rede Pública } & \multicolumn{2}{|c|}{ Rede Privada } & \multirow[t]{2}{*}{$p^{* *}$} \\
\hline & $\mathrm{n}$ & $\%$ & $\mathrm{n}$ & $\%$ & $\mathrm{n}$ & $\%$ & \\
\hline Sexo & & & & & & & 0,744 \\
\hline Masculino & 401 & 41,1 & 265 & 41,7 & 136 & 39,9 & \\
\hline Feminino & 574 & 58,9 & 370 & 58,3 & 204 & 60,1 & \\
\hline Escolaridade da mãe (anos)* & & & & & & & $<0,001$ \\
\hline$<8$ & 257 & 26,4 & 243 & 38,4 & 14 & 04,3 & \\
\hline$>8$ & 715 & 73,6 & 390 & 61,6 & 325 & 95,7 & \\
\hline Sobrepeso/obesidade da mãe* & & & & & & & $<0,001$ \\
\hline Não & 472 & 54,8 & 260 & 47,8 & 212 & 67,0 & \\
\hline Sim & 390 & 45,2 & 285 & 52,2 & 105 & 33,0 & \\
\hline Renda mensal familiar $(\mathrm{R} \$)^{*}$ & & & & & & & $<0,001$ \\
\hline $1^{\circ}$ tercil $(<1500)$ & 269 & 32,5 & 251 & 45,0 & 18 & 06,5 & \\
\hline $2^{\circ}$ tercil (1501-6900) & 271 & 32,7 & 219 & 39,3 & 52 & 19,4 & \\
\hline $3^{\circ}$ tercil $(>7000)$ & 28 & 34,8 & 88 & 15,7 & 200 & 74,1 & \\
\hline Sobrepeso/obesidade do escolar* & & & & & & & 0,439 \\
\hline Não & 676 & 70,3 & 431 & 69,0 & 245 & 72,6 & \\
\hline Sim & 286 & 29,7 & 194 & 31,0 & 92 & 27,4 & \\
\hline \multicolumn{8}{|l|}{ Café da manhã } \\
\hline Frequência/semana* & & & & & & & 0,093 \\
\hline$<4$ & 256 & 27,9 & 180 & 30,6 & 76 & 23,1 & \\
\hline$>5$ & 660 & 72,1 & 409 & 69,4 & 251 & 76,9 & \\
\hline Local* & & & & & & & 0,835 \\
\hline Casa & 845 & 92,7 & 545 & 92,8 & 300 & 92,2 & \\
\hline Escola/outro local & 67 & 7,3 & 42 & 07,2 & 25 & 07,8 & \\
\hline Companhia* & & & & & & & 0,427 \\
\hline Sozinho & 215 & 23,8 & 149 & 25,8 & 66 & 20,3 & \\
\hline Pai e/ou mãe & 428 & 47,5 & 266 & 46,2 & 162 & 49,7 & \\
\hline Outros & 259 & 28,7 & 161 & 28,0 & 98 & 30,0 & \\
\hline \multicolumn{8}{|l|}{ Almoço } \\
\hline Frequência/semana* & & & & & & & 0,907 \\
\hline$<4$ & 161 & 17,6 & 105 & 17,7 & 56 & 17,3 & \\
\hline$>5$ & 756 & 82,4 & 489 & 82,3 & 267 & 82,7 & \\
\hline
\end{tabular}


Caracterização da amostra de escolares de 11 a 14 anos de idade, segundo as variáveis independentes, estratificadas por rede de ensino. Florianópolis, SC, Brasil, 2012/2013.

\begin{tabular}{|c|c|c|c|c|c|c|c|}
\hline \multirow[t]{2}{*}{ Variáveis } & \multicolumn{2}{|c|}{ Total } & \multicolumn{2}{|c|}{ Rede Pública } & \multicolumn{2}{|c|}{ Rede Privada } & \multirow[t]{2}{*}{$p^{* *}$} \\
\hline & $\mathrm{n}$ & $\%$ & $\mathrm{n}$ & $\%$ & $n$ & $\%$ & \\
\hline Local* & & & & & & & 0,041 \\
\hline Casa & 780 & 84,6 & 527 & 87,5 & 253 & 79,0 & \\
\hline Escola/outro local & 142 & 15,4 & 75 & 12,5 & 67 & 21,0 & \\
\hline Companhia* & & & & & & & 0,059 \\
\hline Sozinho & 107 & 11,6 & 84 & 14,1 & 23 & 07,0 & \\
\hline Pai e/ou mãe & 448 & 48,6 & 289 & 48,5 & 159 & 48,6 & \\
\hline Outros & 367 & 39,8 & 222 & 37,4 & 145 & 44,4 & \\
\hline \multicolumn{8}{|l|}{ Jantar } \\
\hline Frequência/semana* & & & & & & & 0,118 \\
\hline$<4$ & 172 & 18,6 & 121 & 20,4 & 51 & 15,5 & \\
\hline$>5$ & 751 & 81,4 & 475 & 79,6 & 276 & 84,5 & \\
\hline Local* & & & & & & & 0,455 \\
\hline Casa & 906 & 97,0 & 596 & 97,4 & 310 & 96,2 & \\
\hline Escola/outro local & 28 & 3,0 & 16 & 02,6 & 12 & 03,8 & \\
\hline Companhia* & & & & & & & 0,162 \\
\hline Sozinho & 52 & 05,6 & 37 & 06,1 & 15 & 04,5 & \\
\hline Pai e/ou mãe & 613 & 66,2 & 411 & 68,4 & 202 & 62,3 & \\
\hline Outros & 261 & 28,2 & 154 & 25,5 & 107 & 33,2 & \\
\hline \multicolumn{8}{|l|}{ Lanches } \\
\hline Frequência/semana* & & & & & & & 0,083 \\
\hline$<4$ & 396 & 43,9 & 272 & 46,8 & 124 & 38,6 & \\
\hline$>5$ & 507 & 56,1 & 309 & 53,2 & 198 & 61,4 & \\
\hline Local* & & & & & & & 0,212 \\
\hline Casa & 586 & 67,3 & 399 & 70,1 & 187 & 62,1 & \\
\hline Escola/outro local & 285 & 32,7 & 171 & 29,9 & 114 & 37,9 & \\
\hline Companhia* & & & & & & & 0,595 \\
\hline Sozinho & 355 & 40,5 & 222 & 39,5 & 133 & 42,2 & \\
\hline Pai e/ou mãe & 240 & 27,4 & 163 & 29,0 & 77 & 24,3 & \\
\hline Outros & 282 & 32,1 & 177 & 31,5 & 105 & 33,5 & \\
\hline
\end{tabular}

$\mathrm{n}=$ total da amostra de acordo com a rede de ensino (pública/privada); *Variáveis com perdas (dados ignorados ou não obtidos); ** Teste qui-quadrado.

Tabela 2

Análise bruta e ajustada da associação entre prevalência de sobrepeso/obesidade e variáveis em escolares de 11 a 14 anos de idade da rede de ensino pública. Florianópolis, SC, Brasil, 2012/2013.

\begin{tabular}{|c|c|c|c|c|c|c|c|}
\hline \multirow[t]{2}{*}{ Variáveis } & \multirow[t]{2}{*}{ Total } & \multicolumn{2}{|c|}{$\begin{array}{l}\text { Sobrepeso/ } \\
\text { Obesidade }\end{array}$} & \multicolumn{2}{|c|}{$\mathbf{R P}_{\text {bruta }}$} & \multicolumn{2}{|c|}{$\mathbf{R P}_{\text {ajustada }}$} \\
\hline & & $\mathrm{n}$ & $\%$ & $($ IC95\%) & $p$ & $($ IC95\%) & $p$ \\
\hline Sexo & & & & & 0,215 & & 0,329 \\
\hline Masculino & 281 & 96 & 34,1 & 1 & & 1 & \\
\hline Feminino & 344 & 99 & 28,8 & $0,8(0,6-1,1)$ & & $0,8(0,5-1,2)$ & \\
\hline Escolaridade da mãe (anos)* & & & & & 0,143 & & 0,232 \\
\hline$<8$ & 242 & 89 & 36,9 & 1 & & 1 & \\
\hline$>8$ & 381 & 105 & 27,5 & $0,7(0,4-1,1)$ & & $0,8(0,5-1,1)$ & \\
\hline
\end{tabular}

*Variáveis com perdas (dados ignorados ou não obtidos).

continua 
Análise bruta e ajustada da associação entre prevalência de sobrepeso/obesidade e variáveis em escolares de 11 a 14 anos de idade da rede de ensino pública. Florianópolis, SC, Brasil, 2012/2013.

\begin{tabular}{|c|c|c|c|c|c|c|c|}
\hline \multirow[t]{2}{*}{ Variáveis } & \multirow[t]{2}{*}{ Total } & \multicolumn{2}{|c|}{$\begin{array}{c}\text { Sobrepeso/ } \\
\text { Obesidade }\end{array}$} & \multicolumn{2}{|c|}{$\mathbf{R P}_{\text {bruta }}$} & \multicolumn{2}{|c|}{$\mathbf{R P}_{\text {ajustada }}$} \\
\hline & & $\mathrm{n}$ & $\%$ & (IC95\%) & $p$ & (IC95\%) & $p$ \\
\hline Sobrepeso/obesidade da mãe* & & & & & 0,001 & & 0,019 \\
\hline Não & 256 & 57 & 22,1 & 1 & & 1 & \\
\hline Sim & 282 & 116 & 41,2 & $1,8(1,3-2,5)$ & & $1,5(1,1-2,1)$ & \\
\hline Renda mensal familiar $(\mathrm{R} \$)^{*}$ & & & & & 0,568 & & 0,986 \\
\hline $1^{\circ}$ tercil $(<1500)$ & 257 & 89 & 34,5 & 1 & & 1 & \\
\hline $2^{\circ}$ tercil $(1501-6900)$ & 208 & 48 & 23,3 & $0,6(0,4-1,1)$ & & $0,8(0,5-1,3)$ & \\
\hline $3^{\circ}$ tercil $(>7000)$ & 83 & 29 & 35,5 & $1,0(0,6-1,5)$ & & $1,1(0,6-1,8)$ & \\
\hline \multicolumn{8}{|l|}{ Frequência das refeições } \\
\hline Café da manhã* & & & & & 0,004 & & 0,001 \\
\hline$<4$ & 174 & 77 & 41,2 & 1 & & 1 & \\
\hline$>5$ & 408 & 114 & 28,0 & $0,6(0,5-0,8)$ & & $0,6(0,4-0,8)$ & \\
\hline Almoço* & & & & & 0,179 & & 0,069 \\
\hline$<4$ & 103 & 27 & 25,9 & 1 & & 1 & \\
\hline$>5$ & 483 & 161 & 33,4 & $1,2(0,8-1,8)$ & & $1,5(0,9-2,4)$ & \\
\hline Jantar* & & & & & 0,098 & & 0,317 \\
\hline$<4$ & 124 & 50 & 40,1 & 1 & & 1 & \\
\hline$>5$ & 464 & 136 & 29,3 & $0,7(0,5-1,1)$ & & $0,8(0,5-1,2)$ & \\
\hline Lanches* & & & & & 0,025 & & 0,313 \\
\hline$<4$ & 258 & 95 & 36,7 & 1 & & 1 & \\
\hline$>5$ & 325 & 84 & 26,8 & $0,7(0,5-0,9)$ & & $0,8(0,5-1,3)$ & \\
\hline \multicolumn{8}{|l|}{ Local das Refeições } \\
\hline Café da manhã* & & & & & 0,885 & & 0,193 \\
\hline Casa & 544 & 165 & 30,4 & 1 & & 1 & \\
\hline Escola/outro local & 35 & 11 & 31,5 & $1,0(0,6-1,7)$ & & $0,7(0,4-1,2)$ & \\
\hline Almoço* & & & & & 0,123 & & 0,022 \\
\hline Casa & 504 & 166 & 33,0 & 1 & & 1 & \\
\hline Escola/outro local & 89 & 19 & 21,1 & $0,6(0,3-1,1)$ & & $0,4(0,2-0,8)$ & \\
\hline Jantar* & & & & & 0,575 & & 0,212 \\
\hline Casa & 588 & 188 & 32,0 & 1 & & 1 & \\
\hline Escola/outro local & 15 & 3 & 21,1 & $0,6(0,1-3,0)$ & & $0,2(0,1-1,7)$ & \\
\hline Lanches* & & & & & 0,559 & & 0,798 \\
\hline Casa & 417 & 138 & 33,1 & 1 & & 1 & \\
\hline Escola/outro local & 144 & 43 & 29,8 & $0,8(0,6-1,3)$ & & $1,0(0,6-1,5)$ & \\
\hline \multicolumn{8}{|l|}{ Companhia nas refeições } \\
\hline Café da manhã* & & & & & 0,084 & & 0,016 \\
\hline Sozinho & 142 & 34 & 23,9 & 1 & & 1 & \\
\hline Pai e/ou mãe & 273 & 82 & 30,0 & $1,2(0,8-1,7)$ & & $1,5(1,0-2,2)$ & \\
\hline Outros & 154 & 60 & 39,0 & $1,6(0,9-2,8)$ & & $2,1(1,2-3,9)$ & \\
\hline Almoço* & & & & & 0,392 & & 0,498 \\
\hline Sozinho & 74 & 26 & 35,3 & 1 & & 1 & \\
\hline Pai e/ou mãe & 284 & 75 & 26,5 & $0,7(0,4-1,2)$ & & $0,5(0,3-0,8)$ & \\
\hline Outros & 229 & 84 & 36,5 & $1,0(0,6-1,5)$ & & $0,7(0,3-1,4)$ & \\
\hline Jantar* & & & & & 0,350 & & 0,238 \\
\hline Sozinho & 32 & 14 & 43,9 & 1 & & 1 & \\
\hline Pai e/ou mãe & 402 & 112 & 27,7 & $0,6(0,3-1,1)$ & & $0,8(0,3-1,9)$ & \\
\hline Outros & 160 & 64 & 40,1 & $0,9(0,4-1,6)$ & & $1,3(0,5-3,5)$ & \\
\hline
\end{tabular}

*Variáveis com perdas (dados ignorados ou não obtidos). 
Análise bruta e ajustada da associação entre prevalência de sobrepeso/obesidade e variáveis em escolares de 11 a 14 anos de idade da rede de ensino pública. Florianópolis, SC, Brasil, 2012/2013.

\begin{tabular}{|c|c|c|c|c|c|c|c|}
\hline \multirow[t]{2}{*}{ Variáveis } & \multirow[t]{2}{*}{ Total } & \multicolumn{2}{|c|}{$\begin{array}{l}\text { Sobrepeso/ } \\
\text { Obesidade }\end{array}$} & \multicolumn{2}{|c|}{$\mathbf{R P}_{\text {bruta }}$} & \multicolumn{2}{|c|}{$\mathbf{R P}_{\text {ajustada }}$} \\
\hline & & $\mathrm{n}$ & $\%$ & (IC95\%) & $p$ & (IC95\%) & $p$ \\
\hline Lanches* & & & & & 0,746 & & 0,818 \\
\hline Sozinho & 209 & 65 & 31,0 & 1 & & 1 & \\
\hline Pai e/ou mãe & 187 & 68 & 36,2 & $1,1(0,8-1,5)$ & & $1,5(0,7-2,9)$ & \\
\hline Outros & 158 & 46 & 29,0 & $0,9(0,6-1,3)$ & & $0,8(0,5-1,2)$ & \\
\hline
\end{tabular}

*Variáveis com perdas (dados ignorados ou não obtidos).

Tabela 3

Análise bruta e ajustada da associação entre prevalência de sobrepeso/obesidade e variáveis em escolares de 11 a 14 anos de idade da rede de ensino privada. Florianópolis, SC, Brasil, 2012/2013.

\begin{tabular}{|c|c|c|c|c|c|c|c|}
\hline \multirow[t]{2}{*}{ Variáveis } & \multirow[t]{2}{*}{ Total } & \multicolumn{2}{|c|}{$\begin{array}{l}\text { Sobrepeso/ } \\
\text { Obesidade }\end{array}$} & \multicolumn{2}{|c|}{$\mathbf{R P}$ bruta } & \multicolumn{2}{|c|}{$\mathbf{R P}$ ajustada } \\
\hline & & $\mathrm{n}$ & $\%$ & $(\mathrm{IC} 95 \%)$ & $p$ & (IC95\%) & $p$ \\
\hline Sexo & & & & & 0,043 & & 0,247 \\
\hline Masculino & 158 & 57 & 36,2 & 1 & & 1 & \\
\hline Feminino & 179 & 39 & 21,5 & $0,5(0,3-0,9)$ & & $0,7(0,3-1,3)$ & \\
\hline Escolaridade da mãe (anos)* & & & & & 0,589 & & 0,277 \\
\hline$<8$ & 14 & 3 & 19,2 & 1 & & 1 & \\
\hline$>8$ & 322 & 23 & 30,1 & $1,4(0,3-6,4)$ & & $3,7(0,3-4,2)$ & \\
\hline Sobrepeso/obesidade da mãe* & & & & & 0,016 & & 0,018 \\
\hline Não & 210 & 40 & 18,9 & 1 & & 1 & \\
\hline Sim & 105 & 46 & 43,9 & $2,3(1,2-4,4)$ & & $2,2(1,1-4,2)$ & \\
\hline Renda mensal familiar (R\$)* & & & & & 0,362 & & 0,725 \\
\hline $1^{\circ}$ tercil $(<1500)$ & 19 & 7 & 38,1 & 1 & & 1 & \\
\hline $2^{\circ}$ tercil (1501-6900) & 50 & 15 & 30,5 & $0,7(0,4-1,5)$ & & $0,9(0,4-1,8)$ & \\
\hline $3^{\circ}$ tercil $(>7000)$ & 199 & 53 & 26,9 & $0,7(0,3-1,3)$ & & $0,9(0,5-1,5)$ & \\
\hline \multicolumn{8}{|l|}{ Frequência das refeições } \\
\hline Café da manhã* & & & & & 0,037 & & 0,460 \\
\hline$<4$ & 70 & 27 & 38,8 & 1 & & 1 & \\
\hline$>5$ & 255 & 61 & 23,8 & $0,6(0,3-0,9)$ & & $0,6(0,2-2,5)$ & \\
\hline Almoço* & & & & & 0,850 & & 0,359 \\
\hline$<4$ & 62 & 16 & 25,5 & 1 & & 1 & \\
\hline$>5$ & 259 & 71 & 27,5 & $1,1(0,4-2,6)$ & & $0,7(0,3-1,8)$ & \\
\hline Jantar* & & & & & 0,903 & & 0,345 \\
\hline$<4$ & 49 & 13 & 26,2 & 1 & & 1 & \\
\hline$>5$ & 276 & 75 & 27,1 & $1,0(0,5-1,9)$ & & $1,3(0,5-3,3)$ & \\
\hline Lanches* & & & & & 0,334 & & 0,331 \\
\hline$<4$ & 118 & 39 & 32,7 & 1 & & 1 & \\
\hline$>5$ & 201 & 46 & 22,3 & $0,6(0,3-1,5)$ & & $0,7(0,3-1,6)$ & \\
\hline \multicolumn{8}{|l|}{ Local das Refeições } \\
\hline Café da manhã* & & & & & 0,812 & & 0,385 \\
\hline Casa & 304 & 82 & 27,0 & 1 & & 1 & \\
\hline Escola/outro local & 19 & 6 & 31,3 & $1,1(0,2-4,5)$ & & $2,3(0,6-7,9)$ & \\
\hline
\end{tabular}


Análise bruta e ajustada da associação entre prevalência de sobrepeso/obesidade e variáveis em escolares de 11 a 14 anos de idade da rede de ensino privada. Florianópolis, SC, Brasil, 2012/2013.

\begin{tabular}{|c|c|c|c|c|c|c|c|}
\hline \multirow[t]{2}{*}{ Variáveis } & \multirow[t]{2}{*}{ Total } & \multicolumn{2}{|c|}{$\begin{array}{l}\text { Sobrepeso/ } \\
\text { Obesidade }\end{array}$} & \multicolumn{2}{|c|}{$\mathbf{R P}_{\text {bruta }}$} & \multicolumn{2}{|c|}{$\mathbf{R P}_{\text {ajustada }}$} \\
\hline & & $\mathrm{n}$ & $\%$ & (IC95\%) & $p$ & (IC95\%) & $p$ \\
\hline Almoço* & & & & & 0,883 & & 0,499 \\
\hline Casa & 235 & 65 & 27,5 & 1 & & 1 & \\
\hline Escola/outro local & 83 & 22 & 26,5 & $0,9(0,5-1,6)$ & & $0,7(0,2-2,4)$ & \\
\hline Jantar* & & & & & 0,442 & & 0,442 \\
\hline Casa & 310 & 84 & 26,9 & 1 & & 1 & \\
\hline Escola/outro local & 10 & 2 & 17,1 & $0,6(0,1-2,2)$ & & $1,7(0,2-3,5)$ & \\
\hline Lanches* & & & & & 0,764 & & 0,753 \\
\hline Casa & 183 & 49 & 27,0 & 1 & & 1 & \\
\hline Escola/outro local & 115 & 28 & 24,3 & $0,9(0,4-1,8)$ & & $0,7(0,3-1,4)$ & \\
\hline \multicolumn{8}{|l|}{ Companhia nas refeições } \\
\hline Café da manhã* & & & & & 0,232 & & 0,229 \\
\hline Sozinho & 61 & 20 & 32,2 & 1 & & 1 & \\
\hline Pai e/ou mãe & 166 & 49 & 29,5 & $0,9(0,4-2,0)$ & & $1,0(0,4-2,1)$ & \\
\hline Outros & 97 & 21 & 21,9 & $0,6(0,3-1,3)$ & & $0,6(0,3-1,3)$ & \\
\hline Almoço* & & & & & 0,685 & & 0,583 \\
\hline Sozinho & 26 & 6 & 24,1 & 1 & & 1 & \\
\hline Pai e/ou mãe & 144 & 42 & 29,3 & $1,2(0,4-3,3)$ & & $1,2(0,4-3,4)$ & \\
\hline Outros & 154 & 38 & 24,5 & $1,0(0,3-3,3)$ & & $1,3(0,3-5,1)$ & \\
\hline Jantar* & & & & & 0,291 & & 0,820 \\
\hline Sozinho & 17 & 8 & 46,1 & 1 & & 1 & \\
\hline Pai e/ou mãe & 189 & 54 & 28,6 & $0,6(0,2-1,7)$ & & $0,7(0,2-2,4)$ & \\
\hline Outros & 115 & 25 & 22,0 & $0,4(0,1-1,8)$ & & $0,9(0,2-4,1)$ & \\
\hline Lanches* & & & & & 0,883 & & 0,288 \\
\hline Sozinho & 132 & 39 & 29,4 & 1 & & 1 & \\
\hline Pai e/ou mãe & 66 & 13 & 19,7 & $0,6(0,2-1,6)$ & & $1,0(0,2-3,5)$ & \\
\hline Outros & 114 & 33 & 28,7 & $0,9(0,5-1,8)$ & & $1,8(0,8-3,8)$ & \\
\hline
\end{tabular}

*Variáveis com perdas (dados ignorados ou não obtidos).

\section{Discussão}

Na presente investigação foram verificadas associações estatisticamente significantes entre o sobrepeso/obesidade dos escolares da rede pública com as variáveis: sobrepeso/obesidade da mãe, maior frequência semanal de café da manhã, realização do almoço na escola/outro local e companhia de outras pessoas nas refeições. Entre os escolares da rede privada observou-se associação significativa apenas com o sobrepeso/obesidade da mãe.

Observou-se elevada prevalência de sobrepeso/ obesidade, de cerca de $30 \%$, na população estudada. Esses níveis de prevalência são comparáveis aos encontrados em estudos conduzidos em outros países, como o Irã $(29 \%),{ }^{11}$ Peru $(33,7 \%$ de sobrepeso e $14,4 \%$ de obesidade) 13 e no Brasil.22-24 Tais níveis de prevalências são preocupantes, uma vez que adolescentes com sobrepeso/obesidade têm maior risco de apresentar doenças crônicas (doenças cardiovasculares, câncer, diabetes e asma) na idade adulta. ${ }^{25}$

Em relação ao sexo, este estudo não detectou diferenças na prevalência de sobrepeso/obesidade entre meninas e meninos de ambas as redes de ensino. Este resultado é distinto do observado em um estudo realizado em Florianópolis, no qual se constatou mais chances de sobrepeso/obesidade entre as meninas de escolas públicas. ${ }^{23} \mathrm{Em}$ outro estudo conduzido na cidade de Fortaleza, observouse prevalência de sobrepeso/obesidade mais elevada entre os meninos de escolas privadas quando comparada aos da escola pública, não se tendo observado diferenças entre as escolas públicas e privadas quanto à prevalência de sobrepeso/obesidade entre as meninas. 22 
A escolaridade materna e a renda familiar não estiveram associadas ao sobrepeso/obesidade de escolares em ambas as redes de ensino, sendo esse dados concordantes com os de outros estudos. 6,26 Por outro lado, estes resultados não estão de acordo com os estudos conduzidos por Fernandes et al.,7 no Brasil, e de Shafaghi et al., ${ }^{11}$ no Irã, que observaram maior risco de sobrepeso/obesidade entre adolescentes cujas mães possuíam maior nível de escolaridade. Nossos achados sugerem que o sobrepeso/ obesidade do escolar parece se distribuir em todos os segmentos de renda mensal familiar na área de estudo, porém ainda há controvérsias quanto à influência do nível socioeconômico familiar sobre o sobrepeso/obesidade de adolescentes.

O estado nutricional da mãe esteve associado ao sobrepeso/obesidade nos escolares em ambas as redes de ensino, sendo este resultando semelhante ao encontrado em outros estudos. 6,7,11,23,27 Estes resultados podem estar relacionados ao fato de que a mãe constitui um exemplo ou referência para os seus filhos e que estas influenciam a formação dos hábitos de vida dos seus filhos, incluindo hábitos alimentares.6,7,27 Esses dados ressaltam a importância da participação dos pais em ações de promoção da saúde e prevenção do sobrepeso /obesidade em adolescentes.

Concordando outros estudos, 2,14 a menor frequência de realização do café da manhã esteve associada à maior ocorrência de sobrepeso/obesidade entre os estudantes da rede pública. Esses dados reforçam as evidências sobre a importância da desta refeição na proteção de sobrepeso/obesidade, particularmente nas classes econômicas menos favorecidas. Uma possível explicação para a omissão dessa refeição seria a preocupação com a imagem corporal e, consequentemente, a realização de dietas restritivas sem orientação profissional. 14 Outra explicação plausível seria a falta de tempo no seu preparo e consumo antes de ir para a escola. ${ }^{2}$ Acrescenta-se, ainda, que existe certa influência da família na promoção dos jovens em realizar o café da manhã, $28-29$ devendo ser utilizadas estratégias de incentivo aos pais sobre a importância da realização desta refeição com seus filhos.

Constatou-se que a realização do almoço na escola/outro local constituiu um fator de proteção para a ocorrência de sobrepeso/obesidade entre os estudantes da rede pública. Esse resultado pode ser explicado pelo fato destes escolares consumirem alimentos com elevado valor calórico em casa, sem supervisão adequada dos pais, ao contrário das escolas públicas, onde o cardápio é usualmente administrado por nutricionistas. No entanto, em estudo realizado no México, constatou-se que escolares que se alimentavam fora de casa tinham mais disponibilidade de acesso e de consumo de alimentos com alta densidade energética, isso poderia contribuir para o desenvolvimento de sobrepeso/ obesidade. ${ }^{3}$

A companhia durante a realização do café da manhã mostrou associação estatística significativa com o aumento da prevalência de sobrepeso/obesidade na rede pública. Esse achado demonstra que o conhecimento sobre alimentação saudável pode influenciar nas escolhas alimentares e consequentemente no ganho de peso, portanto, sugere-se que as escolas públicas realizem mais trabalhos de educação nutricional para os escolares e seus familiares. Em relação à companhia durante as refeições, alguns estudos relataram forte influência dos pais no comportamento alimentar dos filhos.4,6,10-11,28-29 Conforme metanálise realizada por Hammons e Fiese, ${ }^{4}$ a realização de refeições em família, três ou mais vezes por semana reduz $12 \%$ a ocorrência de sobrepeso. De acordo com Costa et al.10 a família auxilia o adolescente desde a escolha dos alimentos até o seu preparo. Cabe ressaltar ainda, que os adolescentes também podem ser influenciados de forma negativa pelo seu grupo de amigos, optando por consumirem alimentos com alta densidade energética (junk food), associados ao desenvolvimento de sobrepeso/obesidade. 30

Entre as limitações desse estudo pode-se destacar o alto percentual de perdas. As diferenças encontradas na distribuição de frequência da idade, sexo e na rede de ensino entre avaliados e não avaliados sugerem a possível ocorrência de viés de seleção. No entanto, reitera-se que o estudo foi realizado em uma amostra representativa dos escolares e atingiu o tamanho inicialmente calculado. Além disso, o poder do estudo de $80 \%$ foi suficiente para detectar razão de prevalência de risco de 1,5 a 2,6 e de proteção de 0,4 a 0,7 . Adicionalmente, ressalta-se que com intuito de corrigir parcialmente o possível viés ocasionado pelas perdas, no processo de construção do banco de dados e definição do peso amostral também foi considerado o percentual de respondentes em relação ao total de elegíveis. Ao mesmo tempo ressalta-se que em pesquisas conduzidas em adolescentes, as taxas de adesão são muitas vezes inferiores àquelas verificadas com outros grupos etários da população $0^{5,15,16}$ e chama-se a atenção para o rigor metodológico com que o estudo foi conduzido.

Conclui-se que a prevalência de sobrepeso/ obesidade nos adolescentes investigados foi elevada e associou-se ao sobrepeso/obesidade da mãe, 
frequência, local e companhia nas refeições entre os escolares na rede pública. Na rede privada somente o sobrepeso/obesidade da mãe associou-se ao sobrepeso/obesidade dos escolares. Desta forma, sugere-se que sejam realizados mais estudos sobre essa temática, destacando sua importância para a elaboração de políticas públicas voltadas para a promoção de hábitos alimentares saudáveis na adolescência, tanto no ambiente familiar quanto escolar, como para todos os segmentos socioeconômicos da população.

\section{Referências}

1. Hoare E, Skouteris H, Fuller-Tyszkiewicz M, Millar L, Allender S. Associations between obesogenic risk factors and depression among adolescents: a systematic review. Obes Rev. 2014; 15 (1): 40-51.

2. Wate JT, Snowdon W, Millar L, Nichols M, Mavoa H, Goundar R, Kama A, Swinburn B. Adolescent dietary patterns in Fiji and their relationships with standardized body mass index. Int J Behav Nutr Phys Act. 2013; 10 (45) $1-12$.

3. Shamah-Levy TS, Nasu LC, Humarán IMG, Aguilar AJ, Ramírez AJM, Villalpando S. La obesidad en niños mexicanos en edad escolar se asocia con el consumo de alimentos fuera del hogar: durante el trayecto de la casa a la escuela. Arch Latino-am Nutr. 2011; 61 (3): 288-95.

4. Hammons AJ, Fiese BH. Is frequency of shared family meals related to the nutritional health of children and adolescents? Pediatr. 2011; 127 (6): e1565-74.

5. Castilho SD, Nuccil LB, Hansen LO, Assuino SR Prevalência de excesso de peso conforme a faixa etária em alunos de escolas de Campinas, SP. Rev Paul Pediatr. 2014; 32 (2): 200-6.

6. Bernardo CO, Vasconcelos FAG. Association of parents' nutritional status, and sociodemographic and dietary factors with overweight/obesity in schoolchildren 7 to 14 years old. Cad Saúde Pública. 2012; 2: 291-304.

7. Fernandes RA, Christofaro DG, Cardoso JR, Ronque ER Freitas Júnior IF, Kawaguti SS, Moraes AC, Oliveira AR. Socioeconomic status as determinant of risk factors for overweight in adolescents. Ciênc Saúde Coletiva. 2014; 16 (10): 4051-57.

8. WHO (World Health Organization). Health behaviour in school-aged children - (HBSC). International Report from the 2005/2006 Survey. Health policy for children and adolescents: Inequalities in young people's health: Copenhagen; 2008. [acesso em 29 Nov 2014]. http://www.euro.who.int/_data/assets/pdf_file/0005/53852 /E91416.pdf

9. Rodrigues EM, Boog MCF. Problematização como estratégia de educação nutricional com adolescentes obesos. Cad Saúde Pública. 2006; 22 (5): 923-31.

10. Costa ALF, Duarte DE, Kuschnir MCC. A família e o comportamento alimentar na adolescência. Adolesc Saúde 2010; 7 (3): 52-8.

\section{Agradecimentos}

Ao CNPq por ter financiado esta pesquisa (Processo $\mathrm{n}^{\circ}$ 483955/2011-6). Aos pais, escolares e escolas por aceitarem participar da pesquisa. À Coordenação de Aperfeiçoamento de Pessoal de Nível Superior pelo financiamento das bolsas de mestrado das acadêmicas Gisele Liliam D'Avila e Priscila Schramm Gonsalez.

11. Shafaghi K, Shariff ZM, Taib MN, Rahman HA, Mobarhan MG, Jabbari H. Parental body mass index is associated with adolescent overweight and obesity in Mashhad, Iran. Asia Pac J Clin Nutr. 2014; 23 (2): 225-31.

12. Li P Yang F, Xiong F, Huo T, Tong Y, Yang S, Mao M. Nutritional status and risk factors of overweight and obesity for children aged 9-15 years in Chengdu, Southwest China. BMC Public Health. 2012; 12: 636.

13. Lozano-Rojas G, Cabello-Morales E, Hernádez-Diaz H, Loza-Munarriz C. Prevalência de sobrepeso y obesidad en adolescentes de un distrito urbano de Lima, Perú 2012. Rev Peru Med Exp Salud Publica. 2014; 31 (3): 494-500.

14. Trancoso SC, Cavalli SB, Proença RPC. Café da manhã: caracterização, consumo e importância para a saúde. Rev Nutr. 2010; 23 (5): 859-69.

15. Motter AF, Vasconcelos FAG, Correa EM, Andrade DF. Pontos de venda de alimentos e associação com sobrepeso/obesidade em escolares de Florianópolis, Santa Catarina, Brasil. Cad Saúde Pública. 2015; 31 (3): 620-32.

16. Assis MAA, Cachera MFR, Grosseman S, Vasconcelos FA, Luna ME, Calvo MC, Barros MV, Pires MM, Bellisle F. Obesity, overweight and thinness in schoolchildren of the city of Florianopolis, Southern Brazil. Eur J Clin Nutr. 2005; 59: 1015-21.

17. Habicht JP. Standardization of quantitative epidemiological methods in the field. Bol Ofic Sanit Panamer. 1974; 76 (5): $375-84$

18. Lohman TG, Roche AF, Martorell R. Anthropometric Standardization Reference Manual. Champign, Illinois: Human Kinetics Books, 1991. P. 44-5.

19. Onis M, Onyango AW, Borghi E, Siyam A, Nishida C, Siekmann J. Development of a WHO growth reference forschool-aged children and adolescents. Bull World Health Organ. 2007; 85 (9): 660-7.

20. WHO (World Health Organization). Physical status: the use and interpretation of anthropometry. Geneva; 1995. 452 p. (Technical Report Series, 854).

21. Araki EL, Philippi ST, Martinez MF, Estima CCP, Leal GVS, Alvarenga MS. Padrão de refeições realizadas por adolescentes que frequentam escolas técnicas de São Paulo. Rev Paul Pediatr. 2011; 29 (2): 164-70. 
22. Campos LA, Leite AJM, Almeida PC. Prevalência de sobrepeso e obesidade em adolescentes escolares do município de Fortaleza, Brasil. Rev Bras Saúde Matern Infant. 2007; 7 (2): 183-90.

23. Benedet J, Assis MAA, Calvo MCM, Andrade DF. Excesso de peso em adolescentes: explorando potenciais fatores de risco. Rev Paul Pediatr. 2013; 31 (2): 172-81.

24. Schmitz BAS, Corso ACT, Caldeira GV, Gimeno SGA, Gabriel CG, Vasconcelos FAG. Overweight and obesity related factors in schoolchildren in Santa Catarina State, Brazil. Arch Latino-am Nutr. 2010; 60 (4): 332-9.

25. Wang YC, McPherson K, Marsh T, Gortmaker SL, Brown M. Health and economic burden of the projected obesity trends in the USA and the UK. Lancet. 2011; 378 (9793): 815-25.

26. Troncon JK, Gomes JP, Guerra-Júnior G, Lalli CA. Prevalência de obesidade em crianças de uma escola pública e de um ambulatório geral de Pediatria de hospital universitário.Rev Paul Pediatr. 2007; 25: 305-10.
27. Marins VM, Almeida RM, Pereira RA, Azevedo Barros MB. The relationship between parental nutritional status and overweight children/adolescents in Rio de Janeiro, Brazil. Public Health. 2004; 118 (1): 43-9.

28. Pearson N, Biddle SJH, Gorely T. Family correlates of fruit and vegetable consumption in children and adolescents: a systematic review. Public Health Nutr. 2008; 18: 1-17.

29. Young KM, Northern JJ, Lister KM, Drummond JA, O'Brien WH. A meta-analysis of family-behavioral weightloss treatments for children. Clin Psychol Rev. 2007; 27 240-9.

30. Payab M, Kelishadi R, Qorbani M, Motlagh ME, Ranjbar SH, Ardalan G, Zahedi H, Chinian M, Asayesh H, Larijani B, Heshmat R. Associação entre o consumo de junk food e a pressão arterial alta e obesidade em crianças e adolescentes iranianos: o Estudo Caspian-IV. J Pediatr. 2015; 91 (2): 196-205

Recebido em 12 de dezembro de 2014

Versão final apresentada em 30 de junho de 2015

Aprovado em 1 de julho de 2015 